\title{
Acoustic Comparison of PET and Latex Wings for Flapping Micro-Air-Vehicles
}

\author{
Lung-Jieh Yang and Suseendar Marimuthu \\ Mechanical and Electromechanical Engineering \\ Tamkang University \\ Tamsui, New Taipei City, Taiwan \\ Ljyang@mail.tku.edu.tw
}

\begin{abstract}
This paper presents the hyper-elastic Latex film as the new material for flapping wings novelly. Compared to one of the conventional flapping wings using PET, the new Latex wing reduces the noise with $15.93 \mathrm{~dB}$ at least. Both wings have a wingspan of $20 \mathrm{~cm}$ and work at the flapping frequency of $13.3 \mathrm{~Hz}$ The leading edge spars and the stiffened ribs of both wings are made of carbon fiber. This newly developed Latex wing is believed to be useful to the application of stealth micro-airvehicle (MAV) in the future.
\end{abstract}

Keywords—micro-air-vehilce (MAV); Latex; noise reduction

\section{INTRODUCTION}

The flapping micro-air-vehicle (MAV) has been of great interest to unmanned-air-vehicle (UAV) fraternity due to their low speed flight, promising maneuverability in the turbulence dominated low Reynolds number regime and sub-centimeter miniaturization prospects. Over the several years of developmental research, most systems, both in the hardware and control sides, have had improvements. But the noise reduction issue of flapping MAVs is seldom discussed and relatively not experimented upon much, given their enormous impact on the generation of critical aerodynamic forces.

In the past, synthetic polymers primarily of plastic nature have been the popular choice for the wings. But their rigidity and directional stability in the microscopic scales gives rise to sharp noises during operation. It is spread over the higher end of the audible range making them heard even over relatively long distances. Moreover, with the passage of time, the plastic wings develop more creases making them prone to generating noise [1]. From the past experience, it is also noted that plastic wings undergo permanent deformation such as folds from to fatigue, and need timely replacement to maintain flight characteristics.

The newly developed Latex wing is a viable solution for the noise issue and is believed to be useful to the application of stealth MAVs in the future. Flexible wings of the right nature could both satisfy aerodynamic needs with a lower acoustic footprint [2].

\section{DESIGN AND FABRICATION}

Before introducing the noise reduction technique in this paper, the authors would like to address the wing actuation prinicple. Similar to the previous works of [3-4], the actuation force or torque available for the flapping wings is drained from the gear-reduction transmission module coupled to a high-speed DC motor powered by a tiny poly-lithium battery. A $7 \mathrm{~mm}$-diameter electrical motor is selected. A four-bar linkage design was also selected as the mechanism for the dynamic flapping of one pair of wings. The exploding view diagram of this gear-reduction transformation module in shown in Fig. 1 [3-4]. The maximum flapping frequency of the flapping wings using this transmission module can be up to $20 \mathrm{~Hz}$

\section{A. Noise reduction issue of MAVS}

Noise reduction issue is important to MAVs subject to the applications of military surveillance and ecological observation domains where covertness contributes to the success of a mission. The noise majorly comes from wing flapping, mechanism operation, and the background noise. In this work the authors focused on addressing the acoustic effect from the wing flapping only and the latter two parts of noise will be filtered. From the literature, the conventional wing materials for flapping MAVs may be PET [3-4], Mylar [5], polyimide [6] parylene [7], etc. The authors have some experience in using PET to flapping MAVs [3-4]. Commercial PET film has good uniformity in thickness and material quality but also has drawback in long-term reliability in mechanical strength. The acoustic noise generated from PET wing is also great.

Herein we use the hyper-elastic Latex film as the new material of flapping wings. The most recent version of the 20$\mathrm{cm}$ wing span flapping MAV 'Golden Snitch' designed and developed at Tamkang University, Taiwan [3-4] was fitted with a $20 \mu \mathrm{m}$ wing made of synthetic Latex. Two spars with an angular separation of $30^{\circ}$ were added on the ventral side of the wing for added mechanical support.

For the sake of comparison, a PET wing of the same dimensions, with a thickness of $25 \mu \mathrm{m}$ was created. 


\section{B. Wing configuration}

Compared to one of the conventional flapping wings using PET [3-4], the new Latex wing will be proved to be superior in noise reduction. The flapping wings shown in Fig. 2 have a wingspan of $20 \mathrm{~cm}$ and work at the flapping frequency of 13.3 $\mathrm{Hz}$. The leading edge spars and the stiffened ribs of both wings are made of carbon fiber. This newly developed Latex wing is believed to be useful to the application of stealth MAVs in the future.

\section{ACOUSTIC MEASUREMENT}

Adobe Audition is used to analyze the audio signals from flapping wings. The process of obtaining spectral waveform of the PET and Latex films were done in following steps.

A. The mechanism was allowed to run at $13.3 \mathrm{~Hz}$ and the sound of the motor combined with the ambient noise was recorded.

B. This recording was clipped to a 3 second audio and was taken as the REFERENCE file for noise reduction profile, used for cancelling mechanism and ambient noise from other videos.

- Audio of the $13.3 \mathrm{~Hz}$ flapping was recorded from the testing platform in Fig. 2 mounted with PET film and Latex wing.

\section{PET film}

- To obtain the sound of only PET film, the noise reduction profile created earlier was subtracted from the PET audio.

- Clear waveform with only the sound of PET film (without mechanism and ambient noise) was obtained as Fig. 3.

\section{Latex film}

- As done in the PET film, the noise reduction profile was used to obtain the sound of only Latex film (without mechanism and ambient noise. The waveform of pure Latex wing is shown in Fig. 4.

\section{FREQUENCY CHARACTERISTICS}

A digital strobe test was performed on the PET and the Latex wing to quantify the frequency characteristics. This test was done in order to measure the frequency of the wing flapping at a fixed voltage. The PET wing operated at $16.7 \mathrm{~Hz}$ at $3.7 \mathrm{~V}$, with the proprietary mechanism used in the Golden snitch MAV. At the same voltage, the Latex wing operated at an elevated frequency of $21.7 \mathrm{~Hz}$.

\section{RESUlt AND CONCLUSION}

- Latex wings (-27.99 dB) have a much smaller acoustic footprint compared to PET wings $(-12.06 \mathrm{~dB})$; the substantial noise reduction is $15.93 \mathrm{~dB}$.

- The peak amplitude of the mechanism alone is -21.63 $\mathrm{dB}$ and with inclusion of Latex film, it is $-11.09 \mathrm{~dB}$. The wings hence have a damping effect on the mechanism noise.

- It is important to note that the frequency above $2 \mathrm{kHz}$ is majorly suppressed and this implies that, if the mechanism noise is successfully suppressed, the Latexwings would be barely heard.

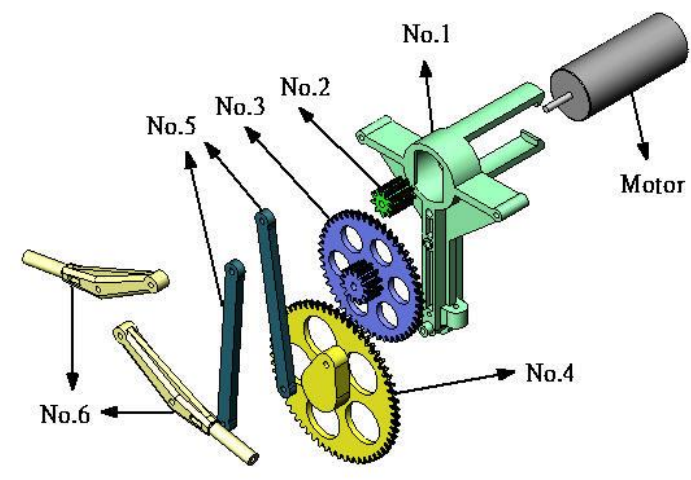

(a)

(b)

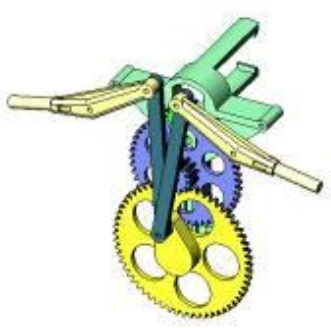

(c)

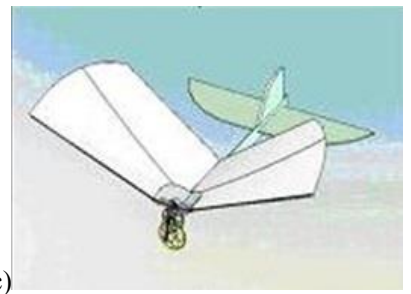

Fig. 1. The configuration of flapping MAV in this work: (a) the exploding view of flapping mechanism; (b) the assebled mechanism; (c) the MAV.

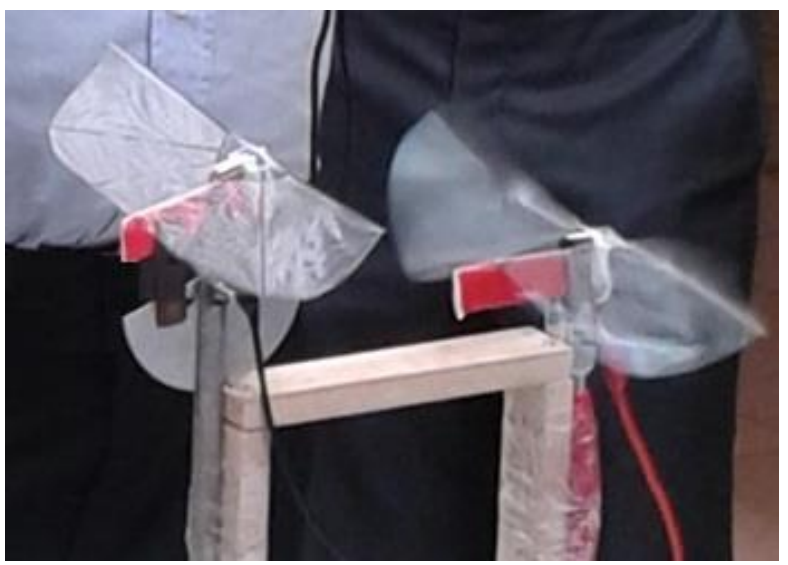

Fig. 2. Flapping wings made of Latex (left) and PET (right); both mounted on the testing platform. 


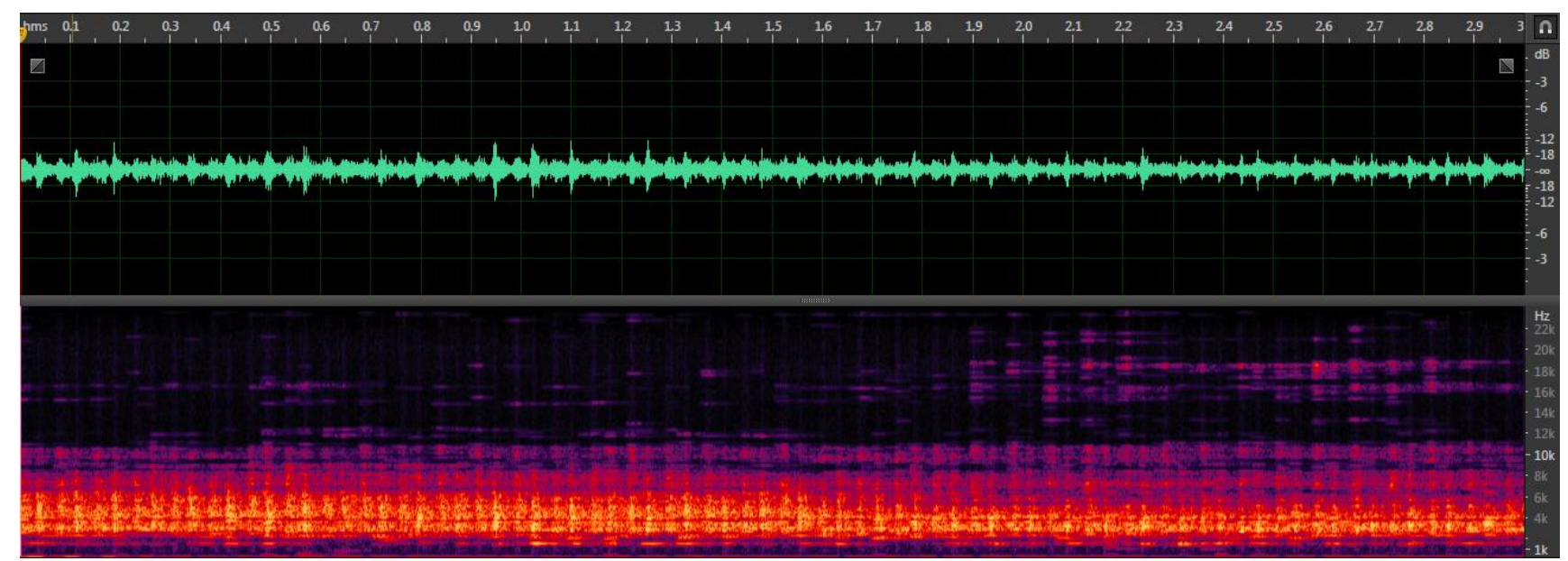

Fig. 3. Spectrum wave form of the PET wing flapping (peak amplitude $=-12.06 \mathrm{~dB}$ ).

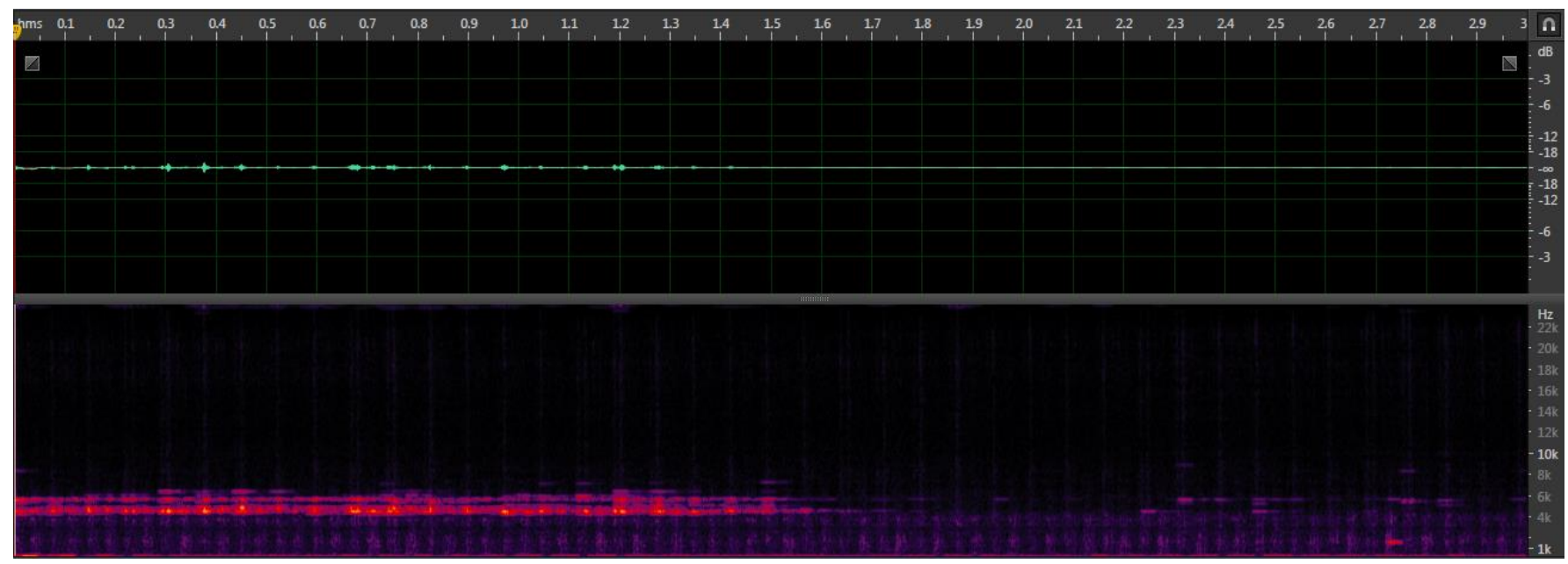

Fig. 4. Spectrum wave form of the Latex wing flapping (peak amplitude $=-27.99 \mathrm{~dB}$ ).

\section{ACKNOWLEDGMENT}

The author appreciates the financial support from the Ministry Of Science and Technology (MOST) of Taiwan with the research project numbers of 101-2623-E-032-001-MY3 and 103-2221-E-032-034.

\section{REFERENCES}

[1] M. Kramer, "On the noise of crumpled candy paper", $139^{\text {th }}$ Meeting Lay language papers, Acoustic Society of America

[2] W. Shyy, M. Berg, and D. Ljungqvist, "Flapping and flexible wings for biological and micro air vehicles," Progress in Aerospace Science, vol. 35, pp. 455-505, 1999.

[3] L. J. Yang, "The micro-air-vehicle Golden Snitch and its figure-of-8 flapping," Journal of Applied Science and Engineering, vol. 15, pp. $197-$ $212,2012$.
[4] L.-J. Yang, A.F. Kuo, and C.K. Hsu, "Wing stiffness on light flapping micro aerial vehicles," Journal of Aircraft, vol. 49(2), pp. 423-431, 2012

[5] M. Keennon, K. Klingebiel, H. Won, and A. Andriukov, "Development of the Nano Hummingbird: a tailless flapping wing micro air vehicle," AIAA Paper, 2012-0588.

[6] J. H. Ko, J. Kim, J. Hong, Y. Yoo, Y. Lee, T. L. Jin, H. C. Park, N. S. Goo, and D. Byun, "Micro/nanofabrication for a realistic beetle wing with a superhydrophobic surface," Bioinspiration \& Biomimetics, vol. 7, no. 1, 016011, 2012.

[7] S. Ho, H. Nassef, N. Pornsinsirirak, Y.-C. Tai, and C.-M. Ho, "Unsteady aerodynamics and flow control for flapping wing flyers," Progress in Aerospace Science, vol. 39, pp. 635-681, 2003.

[8] H. Takahashi, K. Matsumoto, and I. Shimoyama, "Measurement of differential pressure on a butterfly wing," IEEE MEMS 2010, pp. 63-66, Hong Kong, Jan. 24-28, 2010.

[9] N. Pornsinsirirak, M. Liger, Y.-C. Tai, S. Ho, and C.-M. Ho, "Flexible parylene-valved skin for adaptive flow control," IEEE MEMS 2002, pp. 101-104, Las Vegas, Jan. 20-24, 2002. 